\title{
Über (Un-)Möglichkeiten, hiesige Stadtforschung zu postkolonialisieren
}

\author{
Stephan Lanz \\ Über (Un-)Möglichkeiten, \\ hiesige Stadtforschung zu \\ postkolonialisieren \\ Kommentare von: \\ Shadia Husseini \\ Laura Wenz \\ Kanishka Goonewardena \\ Jin Haritaworn \\ Replik von: \\ Stephan Lanz
}

Stephan Lanz

\begin{abstract}
Ausgehend von der These, dass westliche Stadttheorie auf eurozentrischen Prämissen gründet, die urbane Modernität an westliche Städte koppeln und Städte anderswo dem Entwicklungsparadigma einer nachholenden Modernisierung unterwerfen, argumentiert der Beitrag für die Notwendigkeit, die urban studies zu postkolonialisieren. An zwei Beispielen werden Möglichkeiten diskutiert, theoretische und methodische Ansätze einer Stadtforschung voranzutreiben, die postkoloniale Kritik reflektieren. Zum einen erfolgt ein Versuch, Theorien aus, dem Süden' anzuwenden, um etablierte Wahrheiten in der Stadt des globalen Nordens zu erschüttern. Zum anderen wird das internationale Forschungsvorhaben "Global Prayers - Redemption and Liberation in the City" als Versuch diskutiert, im Rahmen eines transregionalen, transdisziplinären und transinstitutionellen Ansatzes einige eurozentrische Fundamente der urban studies zu dekonstruieren und eine kosmopolitischere Stadtforschung voranzutreiben.
\end{abstract}

Ersteinreichung: 18.9.2014; Veröffentlichung online: 10.4.2015

An english abstract can be found at the end of the document.

Die westliche Stadttheorie, so kritisiert Jennifer Robinson (2006), ist maßgeblich von zwei theoretischen Manövern geprägt: Zum einen stellt sie seit Georg Simmel oder Louis Wirth eine selektive Assoziation zwischen Stadt und Modernität her, zum anderen konzipiert ihr „developmentalism“ (ebd.: 4) Städte jenseits des Nordens als unterentwickelt und defizitär. Nur bestimmte Städte im Westen werden so als privilegierte Orte von Erfindungen und Modernität imaginiert, während Städte anderswo auf der Welt als nichtmoderne Orte gelten. Auf sie wird das Paradigma der Entwicklung im Sinne einer nachholenden Modernisierung angewandt. Wie aktuell diese Kritik bezogen auf die deutschsprachige Stadtforschung ist, lässt sich für beide dieser Manöver nachvollziehen.

\section{Die europäische Stadt und der Rest}

So boomen in den letzten Jahren Forschungsvorhaben und Publikationen, die sich - unter anderem im Rahmen eines Programms des Bundesministeriums für Wissenschaft und Forschung (BMBF) - mit sogenannten Megastädten beschäftigen, ohne die aus postkolonialen Machtungleichheiten resultierende „Geopolitik des Wissens“ (Mignolo 2002) zu reflektieren, in die 
jeder Forschungsprozess schon im Rahmen seiner Förder- und Evaluationsbedingungen eingebettet ist (vgl. Kaltmeier 2012). Basierend auf dem Entwicklungsparadigma (vgl. zu dessen Kritik Lossau 2012, Ziai 2012) und mit Blick auf Fragen der Regierungsfähigkeit von,Megastädten “ im globalen Süden zielen solche Programme und Studien langfristig mehrheitlich darauf, modellhafte politische „Lösungsstrategien“ (BMBF 2010) oder, so etwa das Geographische Institut der Universität Köln (o.D.), „Instrumente für Stadtplanung und für städtische Governance-Systeme zu entwickeln und so eine nachhaltigere Entwicklung der im Globalen Süden verorteten Megastädte zu ermöglichen“.[1]

Auf der anderen Seite zeigt das schon länger in der Kritik stehende Konzept der europäischen Stadt (vgl. zuletzt Ha 2014) eine bemerkenswerte Beharrlichkeit. So enthält das 2012 publizierte Handbuch Stadtsoziologie ein Kapitel, in dem „die europäische Stadt“ als „Ort, an dem die moderne Gesellschaft entstanden ist“ (Siebel 2012: 202), in Abgrenzung von „Städten anderer Kulturkreise" (ebd.: 204) zu einem von Emanzipation, Demokratie, Selbstbestimmung und Hoffnung auf besseres Leben bestimmten Idealtypus stilisiert wird. Dabei werden die gewaltsame globale Ausdehnung der ,europäischen Stadt' im Zuge der Kolonialregime, ihre nur mithilfe der Ausbeutung der Kolonien mögliche Industrialisierung sowie die systematische Ausgrenzung ganzer Bevölkerungsgruppen zunächst aus der Bürger- und später aus der kapitalistischen Industriestadt (vgl. Lanz 2008) zu nicht erwähnenswerten Betriebsunfällen degradiert, anstatt sie als strukturelle Bestandteile des „historisch Besonderen der Stadt in Europa“ (Siebel 2012: 204) zu verstehen. Diese Konstruktion ist nur auf der Basis einer essentialistischen Vorstellung von Kulturen als ethno-national homogene ,Kulturkreise‘ möglich, die den Repräsentationscharakter und die grundsätzlich unabgeschlossenen Austauschprozesse kultureller Praktiken übersieht. Sie gründet auf der eurozentrischen Vorstellung der einen Moderne und verkennt, dass sich Moderne in einem „welthistorischen Prozess“ (Comaroff/Comaroff 2012: 16) in alternierenden Pfaden herausbildete und dass sich Vorstellungen von Modernität wandeln (vgl. King 2003). Schließlich gründen Konzepte wie Modernität oder Europa nicht auf objektiven Erkenntnissen, sondern enthalten als Bestandteil des Repräsentationssystems, in dem sie formuliert werden, allenfalls „partielle Wahrheiten“ (Clifford 1986, vgl. auch Costa 2005). Engin Isin (2003) hat längst herausgearbeitet, dass die exklusive Kopplung der Konzepte Bürger und Bürgerschaft an eine europäische Stadt im Kontrast zu einer ,orientalischen Stadt' durch die darin Max Weber folgenden Sozialwissenschaften auf orientalistischen Annahmen gründet. So offenbart sich das Konzept der europäischen Stadt als Bestandteil der von Stuart Hall (1994) als ,der Westen und der Rest 'bezeichneten Diskursformation, die das Westliche als städtisch, modern, zivilisiert und säkularisiert, den ,Rest' hingegen als unterentwickelt, traditionell und religiös deutet (vgl. Lanz 2007).

\section{In Richtung einer postkolonialen Stadttheorie}

Dass im selben Handbuch Stadtsoziologie der Herausgeber Frank Eckardt mit Johanna Hoerning (2012) für eine postkoloniale Stadtsoziologie plädiert, zeugt von einem diskursiven Wandel in der etablierten deutschen Stadtforschung. Beide kritisieren zu Recht ein in den Sozialwissenschaften vorherrschendes 
(bequemes) Verständnis von Postkolonialität als zeitliche Epoche. Sie schließen sich einer postkolonialen Kritik an, die der erste daran orientierte Stadttheoretiker Anthony King ,as an oppositional form of knowledge that critiques Eurocentric conceptions of the world“ definiert (2003: 262). Als Set interdisziplinärer Diskurspraktiken, die fundamental um Fragen von Subjektivität, Macht und Herrschaft kreisen (Roy 2009), beschäftigen sich postkoloniale Ansätze „längst nicht mehr nur mit den Wirkungen der Kolonialisierung, sondern bezieh[en] auch die aktuell bestehenden neokolonialen Machtverhältnisse und die diversen ,kulturellen Formationen', die in Folge von Kolonialisierung und Migration in den Metropolen entstanden sind, in ihre Betrachtungen mit ein" (Castro Varela/Dhawan 2005: 25). Während die Forderung von Eckardt und Hoerning, die westliche Stadtforschung von ihren Fundamenten aus zu dekonstruieren, im Einklang mit der postkolonialen Kritik steht, verharren ihre beispielhaft skizzierten Perspektiven für eine postkoloniale Stadtsoziologie jedoch eng und deskriptiv an direkten Wirkungen des deutschen Kolonialismus und bleiben so hinter ihrer theoretischen Kritik zurück.

Denn eine Kritik der eurozentrischen Wissensproduktion über die Stadt erfordert eine fundamentale Dekonstruktion nicht nur des Konzepts der europäischen Stadt, sondern all seiner tief in die etablierte Stadtforschung eingeschriebenen Prämissen. Dazu gehört gerade in Deutschland deren systematische Weigerung, das gesellschaftliche Macht- und Herrschaftsverhältnis, in dem in der Stadt Fremdes und Eigenes definiert, in ein hierarchisches Verhältnis zueinander gestellt und in eine „praktische Mechanik des Ausschlusses“ (Terkessidis 2004: 99) übersetzt wird, mit postkolonial informierten Rassismustheorien zu analysieren, anstatt mit Integrations- und Multikulturalismusparadigmen zu hantieren. Denn deren unhinterfragte Prämissen basieren auf den „natio-ethno-kulturellen“ Unterscheidungen zwischen ,Wir‘ und ,Nicht-Wir‘ (Mecheril 2004: 14), die in der historischen Tradition einer essentialistischen Konstruktion der deutschen Nation als Kulturgemeinschaft stehen (vgl. Lanz 2007; 2013). Nur so ist es beispielsweise zu erklären, dass regelmäßig aufflackernde Ghettound Parallelgesellschaftsdiskurse oder ein sich formierender ,,antimuslimischer Urbanismus" (Tsianos 2013) nicht als Bestandteile einer historischen Diskursformation erkannt werden. Vielmehr werden sie mit Konzepten wie Ausländer- oder Fremdenfeindlichkeit bearbeitet, die längst einheimische Nachkommen von Einwander_innen immer wieder zu ,Fremden“ machen.

Im Gegensatz zum deutschsprachigen Raum haben postkoloniale Ansätze basierend auf wegweisenden Publikationen etwa von Anthony King (1990), Nezar AlSayyad (1992) oder Jane Jacobs (1996) in den englischsprachigen urban studies zwar schon eine längere Geschichte (einen frühen Überblick gibt Yeoh 2001), sie blieben aber auch dort die längste Zeit eine minoritäre Randerscheinung. Postkoloniale Beiträge der lateinamerikanischen Stadtforschung wurden zudem gänzlich übersehen (vgl. Huffschmid/Wildner 2013). Mit einem kritischen Blick auch auf postkoloniale Wissensproduktionen der urban studies halten es Jennifer Robinson, Ananya Roy oder Aihwa Ong für eine noch zu meisternde Aufgabe, eine „post-colonial urban theory“ (Roy 2014) zu entwickeln. Ong argumentiert, dass die beiden in der kritischen „Western-centric urban theory“ (Edensor/Jayne 2012: 5) vorherrschenden Ansätze zu eng an ihrem 
marxistischen Stammbaum verharren: Der polit-ökonomische Ansatz fokussiere auf den globalen Kapitalismus als „singuläre Kausalität“ für die Produktion von Stadt als Ort der Kapitalakkumulation und subsumiere urbane Heterogenität unter ein minimales Set von Erklärungsmustern (Ong 2011: 6, ähnlich Simone 2011). Offensichtlich zeigt sich dies in der Ignoranz gegenüber der urbanen Bedeutung von Religion (vgl. Lanz 2014a). Die postkoloniale Stadtforschung wiederum arbeitet zwar alternierende urbane Modernitätspfade und subalterne Handlungsmacht heraus. Beide dabei vorherrschenden Perspektiven - der Fokus auf Kontinuitäten der kolonialen Vergangenheit in der urbanen Gegenwart und der Fokus auf die politische Handlungsmacht subalterner Gruppen - verengten aber bei der Analyse unterschiedlichster urbaner Transformationen den Blick auf postkoloniale Subjektivität (vgl. Ong 2011). Roy kritisiert zudem ontologische und topologische Lesarten eines „subaltern urbanism“ (2011a: 235), die Identitäten im „Slum ' der ,Megastadt' essentialisieren oder den unternehmerischen Habitus und die Selbstorganisation der Armen feiern (vgl. auch Lanz 2008).

Auf der Suche nach einem anderen Fokus auf den postkolonialen Urbanismus schlägt Roy daher einen Wechsel vor, „from the postcolonial as an urban condition to the postcolonial as a critical deconstructive methodology" (2011b: 308). Die Zentren und Begriffe urbaner Theorieproduktion sollen zugunsten „new geographies of theory“ (2009) von Euroamerika weg verrückt werden. Mit Dipesh Chakrabarty (2000) sollen neue Theorien das westliche Wissen über die Stadt „provinzialisieren“, das heißt die Partialität seiner Wahrheitsansprüche offenlegen. Zudem sollen sie in ihren Perspektiven globaler und kosmopolitischer sowie in ihren Geltungsansprüchen lokaler situiert und bescheidener sein (vgl. Robinson 2010). Roy und Ong (2011) entwickeln dafür ein auf Gayatri Spivak zurückgehendes Verständnis von worlding, das mit dem kapitalfixierten Zentrum-Peripherie-Modell des Global-City-Ansatzes bricht. Spivak (1999) prägte den Begriff des ,Weltmachens', um nachzuvollziehen, auf welche Weise die koloniale Welt durch die Produktion eines spezifischen Wissens sowie die pure Anwesenheit der Kolonisator_innen hervorgebracht und angeeignet werden konnte. Mit Blick auf die Stadttheorie bezieht sich worlding bei Roy zunächst auf die urbane Wissensproduktion selbst und zielt auf eine Dekonstruktion von deren globalen Wahrheitsregimes. Zudem überträgt sie mit Ong am Beispiel asiatischer Städte das Konzept auf urbane Alltagspraktiken - seien sie dominant oder subaltern, amtlich oder irregulär -, die über existierende urbane Lebensverhältnisse hinausblicken: „[They] creatively imagine and shape alternative social visions and configurations that is ,worlds““ (Roy 2011b: 314). Als „worlding from below“ wiederum hat AbdouMaliq Simone (2001: 16) schon früher die globale Ausdehnung des urbanen Afrika gedeutet. Roy sieht daher als zentrales Anliegen des Projekts einer postkolonialen Stadttheorie ,an analysis of the worlding of the world but equally an effort to imagine other worlds“ (2014: 18).

\section{Mögliche neue Routen einer postkolonialisierten deutschen Stadtforschung}

Die Worlding-Konzepte von Simone und Roy/Ong bilden ein sinnvolles Instrumentarium, um urbane Konfigurationen aus einer globalen Perspektive 
jenseits der klassischen Nord-Süd-Dichotomie zu analysieren (vgl. bspw. Lanz 2014a, Heck/Lanz 2014). Im Folgenden möchte ich aber mithilfe von zwei Beispielen andere mögliche Routen einer postkolonial informierten Stadtforschung skizzieren.

Zum einen versuche ich am Beispiel von zwei zugegebenermaßen naheliegenden urbanen Konstellationen in Berlin, westzentrischen Theoriekonzepten der urban studies eine Strategie entgegenzuhalten, die „etablierte Wahrheiten über die gegenwärtigen Verhältnisse“ mit Blick auf die ,eigenen“ Städte mithilfe von „Theorien aus dem Süden“ zu erschüttern sucht. Diese jüngst von Jean und John Comaroff (2012: 32) erhobene Forderung zielt selbstverständlich nicht darauf, einen hierarchischen Dualismus durch sein Gegenteil abzulösen. Vielmehr geht es darum, eine kritische Verfremdung zu versuchen, um „dem Normalen seine Normalität zu nehmen“(ebd.). Dies folgt nicht zuletzt der Erkenntnis, dass die Effekte weltumspannender Zirkulationsprozesse auch im Westen urbane Konstellationen hervorbringen, die für euroamerikanische Städte entwickelte Konzepte nicht einmal mehr vermeintlich erklären können.

Zum anderen möchte ich den Blick auf Fragen des empirischen doing of urban studies richten, die die theoriefixierten Debatten von Ananya Roy und anderen über eine zu postkolonialisierende Stadtforschung allzu oft ausblenden. Nimmt man den Anspruch ernst, die eurozentrische urbane Wissensproduktion dekonstruieren zu wollen, muss neben den angewandten Theorien auch die Art und Weise kritisch reflektiert werden, wie sich die ,Geopolitik des Wissens' in einem Forschungsprozess spiegelt (vgl. Kaltmeier 2012). Dies gilt von der Akteurskonstellation eines Projekts über die Datenerhebung bis hin zur Repräsentation der Forschungsergebnisse. Mit dieser Absicht werde ich das Projekt „Global Prayers - Redemption and Liberation in the City“ skizzieren, das in meiner Praxis einen ersten solchen Versuch darstellt.

\section{Theorien aus dem Süden in der Stadt des globalen Nordens}

Im September 2012 marschierten Flüchtlinge aus verschiedenen deutschen Städten in einem Verstoß gegen die Residenzpflicht nach Berlin, errichteten auf dem Oranienplatz ein Zeltlager und besetzten wenig später eine leerstehende Schule. Bis zur ihrer Räumung im Frühjahr bzw. Sommer 2014 wohnten mehrere Hundert Flüchtlinge dort und organisierten die Kämpfe ihres refugee strike für Rechte auf Aufenthalt, Arbeit, politische Partizipation und einen selbstbestimmten Wohnort (vgl. Aktionskreis 2013). Die heterogen zusammengesetzte Mieterinitiative Kotti \& Co wiederum besetzte im Mai 2012 einen öffentlichen Platz am Kottbusser Tor und errichtete eine gecekondu genannte Protesthütte. Ihren Kampf gegen den drohenden Verlust von Wohnung und Wohnort durch existenzbedrohende Mietsteigerungen und eine verfehlte Wohnpolitik führen sie seither in Demonstrationen, politischen Verhandlungen und Events.

Beide aus diesen Besetzungen öffentlicher Räume entstandenen urbanen Konstellationen sind meines Erachtens mit herkömmlichen Begriffen der sozialwissenschaftlichen Stadtforschung nicht sinnvoll zu greifen.[2] Bezogen auf die postpolitische Situation der gegenwärtigen Stadt (vgl. Lanz 2014b, 
Michel/Roskamm 2013) stellen beide einen Bruch des Gegebenen dar (vgl. Isin 2008). Sie zielen auf eine demokratische „Gleichheitslogik als Anteil der Anteillosen“ (Rancière 2002: 146), entfernen die Subjekte von den ihnen zugewiesenen gesellschaftlichen Orten und repolitisieren so die Stadt.

Mit all den neuen Subjektivitäten und Kämpfen in der globalisierten Stadt so argumentiert Engin Isin (2008) ähnlich wie der mexikanische Stadtforscher Sergio Tamayo (2013) - manifestiert sich citizenship (oder ciudadanía in Lateinamerika) immer weniger als Status und Habitus. Vielmehr erweist sie sich als eine soziale Praxis, bei der sich Subjekte zu citizens aufschwingen, das heißt zu „claimants of justice, rights and responsibilities“ (Isin 2008: 18; vgl. dazu auch Hess/Lebuhn 2015). Dies offenbaren auch die beiden Berliner Initiativen, die sich die Schauplätze ihrer Kämpfe kollektiv aneigneten und sie in temporäre „Ciudadanía-Räume“ transformierten (Tamayo 2013). Ihre „acts of citizenship“ (Isin 2008) formulieren existentielle Ansprüche und verknüpfen sie mit der gesamten Stadt: „Es geht um unsere Existenz. Es geht um diese Stadt. Wir fordern unsere Rechte ein. Es geht um das Recht auf Stadt“ (Kotti \& Co 2012). Als Spiegel der globalisierten Stadt sind beide bezogen auf soziale, ethnische, nationale, religiöse oder politische Zugehörigkeiten „mehr oder weniger zufällig zusammengewürfelt“ (ebd.) und äußerst heterogen. Sie verweigern jede Einteilung in bekannte Kategorien und lehnen normative Konzepte von Multikulturalität oder Integration ab. In ihrem Kampf sei es schwer genug, „normal“ (ebd.) miteinander umzugehen. Dies war besonders in der prekär selbstverwalteten Schule, in der neben den Aktivist_innen andere Asylsuchende aus vielen Nationen, osteuropäische Wanderarbeiter_innen oder zwangsgeräumte Einheimische wohnten, kaum zu meistern.

Folgt man dem postkolonialen Theoretiker Walter Mignolo (2000), der den Kosmopolitismus als Set von Projekten in Richtung eines weltumspannenden Zusammenlebens bezeichnet hat, handelt es sich beim refugee strike um ein kosmopolitisches Projekt par excellence. Gemeint ist aber kein bürgerlich-elitärer, an einen eurozentrisch-liberalen Universalismus gekoppelter Kosmopolitismus, sondern ein „subaltern cosmopolitanism as politics“ (Gidwani 2006). Für Vinay Gidwani besteht dieser aus „practices of thinking, border crossing, and connecting that are transgressive of the established order [...]. Rejecting the sacred and secular motifs of society neither as rights-borne privilege nor as charity, but as irrevocable claim“ (ebd.: 19).

Arjun Appadurai (2001; 2011) bezeichnet die alltäglichen Kämpfe für bürgerschaftliche Rechte in den Armenvierteln von Mumbai als „cosmopolitism from below“ und fasst sie mit dem Konzept der „deep democracy“. Da dort Menschen mit unterschiedlichen Sprachen, Religionen oder Kasten auf engem Raum zusammengewürfelt leben, erfordert ihr Kampf ständige Übersetzungen. Diese müssen die interne kulturelle Diversität und die Kluft zu den Institutionen der offiziellen Stadt überbrücken. Dieser Kosmopolitismus ist keiner der freien Wahl, „it is a cosmopolitanism driven by the exigencies of exclusion rather than by the privileges (and ennui) of inclusion“ (2011: 32).

Ähnlich wie Appadurai versuchen Partha Chatterjee (2004) oder Asef Bayat (2012) mit ihren Konzepten der political society bzw. der ,Straßenpolitik‘ einen widerständigen Aktivismus in Städten Indiens oder des Nahen Ostens zu erfassen, in denen große Bevölkerungsgruppen dem Staat nicht als citizens, sondern als marginalisierte, oft in die Illegalität gedrängte Subjekte 
gegenüberstehen. Da Eliten und Staatsapparate sie nicht als Teil der bürgerlichen Gesellschaft betrachten, stehen ihnen deren Kanäle für politische Partizipation nicht offen. Entsprechend unterscheiden sich ihre politischen Forderungen und ihre oft illegalen Mittel im Kampf um existentielle Rechte fundamental von denen von ,Bürger_innen'. Bayat analysiert die bedeutende Rolle, die dabei die Straße spielt, insofern sie es kaum organisierten Marginalisierten ermöglicht, unmittelbar zu kommunizieren und die eigenen Körper im Konflikt mit den Staatsapparaten einzusetzen.

Ohne hier eine gründliche Analyse vornehmen zu können, weist das Verhältnis zwischen Staat, urbaner Gesellschaft und den um Rechte kämpfenden Flüchtlingen offensichtliche Gemeinsamkeiten mit den Analysen von Appadurai, Chatterjee oder Bayat in Städten des globalen Südens auf. Als selbst bezeichnete „non-citizens“ (Arbeitskreis 2013), die im Zuge einer weltumspannenden Mobilität in einer „Weltrisikogesellschaft“ (Ulrich Beck) aus der Stadt des Nordens nicht wieder verschwinden werden, agieren die Flüchtlinge illegal und verfügen über keinerlei Bürgerstatus, der ihnen Zugang zu zivilgesellschaftlichen Formen politischer Partizipation ermöglichen würde. Sie müssen daher auf andere Mittel zurückgreifen. Gleichwohl stigmatisieren selbst aufgeschlossene Grünen-Politiker_innen die Drohungen der Flüchtlinge mit Hungerstreik und Dachsprung als illegitime Erpressungen. Solchen Einschätzungen liegen eurozentrische, auf den Prämissen der bürgerlichen Gesellschaft basierende Vorstellungen eines legitimen politischen Handelns zugrunde.

Kotti \& Co stellt sich in eine den Flüchtlingen vergleichbare kämpferische Tradition. Ihr gecekondu bezieht sich auf einen selbstorganisierten informellen Städtebau, mit dem ländlicheZZuwander_innen in türkischen Metropolen dem Staat ihr Recht auf die Stadt abgerungen haben. Dies verweist auf übermittelte Erfahrungen in der türkischen Einwanderercommunity, der einige Mitglieder der Initiative angehören.

Mithilfe postkolonial geschulter Konzepte, so meine These, lässt sich die politische Bedeutung von Kotti \& Co und dem refugee strike für Berlin greifen. Ihr ,Kosmopolitismus als Politik‘ widersetzt sich herkömmlichen Kategorisierungen sozialer Gruppen und erprobt unter schwersten Bedingungen, wie im Alltag einer hochgradig heterogenen urbanen Gesellschaft Interessen und Bedürfnisse übersetzt und verhandelt werden können. Ihre acts of citizenship durchbrechen die Ausgrenzungsmuster zivilgesellschaftlicher Partizipationskanäle der bürgerlichen Stadt, um für Anteillose oder in ihren Rechten Gefährdete eine „Logik der Gleichheit als Anteil der Anteillosen“ (Rancière) zu erkämpfen. Trotz ihrer Räumung unter Gewaltandrohung ließen die Flüchtlingsbesetzungen, ihre Unterstützung durch diverse städtische Milieus und die lange Duldung durch die Kommune Kreuzberg Potenziale einer urbanen „democracy without borders“ (Appadurai 2001: 42) zumindest temporär aufscheinen.[3]

\section{Trans...: Eine experimentell vergleichende Stadtforschung}

Das Forschungsvorhaben „Global Prayers - Redemption and Liberation in the City" stellt einen Versuch dar, die Postkolonialisierung der Stadtforschung mit einem transregionalen, transdisziplinären und transinstitutionellen Projekt 
voranzutreiben. Es ging aus dem die Buchreihe metroZones begründenden Ziel hervor, urbanen Alltag jenseits der ,europäischen Civitas zu erforschen (vgl. Becker/Lanz 2003). Die metroZones-Programmatik folgt seit 2003 den Prämissen, dass Städte des Südens und Nordens zum gleichen postkolonialen Analysefeld gehören und dass der urbane Süden als „palimpsests of colonization, de- re- and neo-colonization“ (de Boeck 2002: 244) eigenständigen Modernisierungspfaden folgt. Zudem wurde der herkömmliche Blick urbaner Analysen im Sinne einer „inventive methodology“ (Simone 2010: 279) zugunsten einer Süd-Nord-Perspektive gedreht. Süden und Norden sind dabei keine ontologischen oder grundsätzlich geografischen Kategorien, sondern in Anlehnung an Stuart Halls (1994) Konzept vom Westen und dem Rest relationale, einander bedingende Bestandteile einer einzigen Diskursformation.

Basierend auf diesen Manövern drängten sich weltweit neuartige urbane Manifestationen des Religiösen in das Blickfeld der metroZones-Forschungen. Insofern solche Entwicklungen fundamentalen Annahmen der kritischen Stadttheorie widersprachen, hatten auch unsere eigenen urban studies sie lange übersehen oder als Fundamentalismus urbaner Armer stigmatisiert. Die anfängliche Programmatik von „Global Prayers“ basierte entsprechend auf einer normativen Religionskritik und koppelte den urbanen Boom religiöser Bewegungen eindimensional an soziale Verwerfungen in Folge des globalen Neoliberalismus. Konfrontiert mit postkolonialer Kritik, rangen wir uns erst im Rahmen eines Workshops zu der These durch, dass die dahinter stehende Annahme, urbane Modernität sei per se säkular, als eurozentrische Prämisse der Stadtforschung dekonstruiert werden müsse. Da mir hier der Platz fehlt, um alle Dimensionen des Projekts zu diskutieren (vgl. Lanz 2014a, Becker et al. 2013), möchte ich die strategische Konstellation und zentrale methodische Manöver bezogen auf mögliche Routen, Stadtforschung zu postkolonialisieren, diskutieren.

Die extreme Kluft zwischen urbanem Alltag und urbaner Wissensproduktion legte es nahe, vertraute Ansätze der urban studies beiseitezulegen und einen experimentellen, auf „de-centering“, „de-familializing“ und „untruthing“ (Jacobs 2012: 907) zielenden Forschungsansatz zu konzipieren. In sehr unterschiedlichen Städten weltweit sollten explorative Fallstudien fragend und induktiv vorgehen, um die Bedeutung urbaner Religion als Bestandteil der materiellen, sozialen und symbolischen Produktion des Städtischen und als Artikulation urbaner Modernität zu untersuchen. Die Fallstudien selbst stellten keine klassischen Vergleiche an, sondern folgten einem experimentellen Vergleichsansatz (vgl. Robinson 2010). Im Sinne von Jane Jacobs war „Global Prayers“ ein 1+1+ -Vorhaben: „(+) points to multiplicity, and in the direction of emergence and becoming“ (2012: 905) und „the multiple (1+1) generates an ever-present ground ,un-truthing““ (ebd.: 907). Es ging also weniger darum, Ähnlichkeiten und Unterschiede zwischen abgegrenzten Einheiten zu untersuchen, als darum, Transformationen, Verknüpfungen oder Verschmelzungen urbaner Religions- und religiöser Urbanitätsformen herauszuarbeiten. Mit Fokus auf ihre global/lokalen Manifestationen und Prozesse sowie ihre materiellen und imaginären Netzwerke und Verknüpfungen wurden sie im Sinne von Simone, Roy und Ong als Praktiken des worlding untersucht (vgl. Lanz 2014a). Diese Projektkonstellation folgte der Erkenntnis, dass der Versuch einer 
Dezentrierung als wesentlicher Bestandteil von Postkolonialisierung die Existenz einer Vielfalt von Perspektiven und Analysen voraussetzt; einer Vielfalt, die Evidenz erlangt in „the notion of the center against which one works“ (Jacobs 2012: 904).

Das 1+1+-Konzept von „Global Prayers“ geht über inhaltliche Fragen hinaus und entspricht Colin McFarlanes Verständnis eines Vergleichs als Strategie:

„In the expansive reading of comparison [...], I argue for attention not just to different scholarly knowledges on cities from social science across the world, but different activist and public knowledges that are important for the production of a more global, more democratic urban studies characterized by diverse urban epistemes and imaginaries“ (2010: 727).

Der Vergleich ist so jenseits methodischer Fragen eine Denkweise und ein strategisches Werkzeug zur Konfiguration eines Projekts, das transdisziplinäre, transinstitutionelle und transregionale Überschreitungen vornimmt; ein Instrument „for creating new conversations and collaborations, for reading different traditions and connections, and for expanding the field of critique and inquiry" (ebd.: 730). Ein solcher Vergleich geht über ein gegenseitiges „learning from*“ (so der Titel einer früheren metroZones-Ausstellung, vgl. Becker et al. 2003) hinaus: Er ist „a key site for the urban imagination - a potential site of politics“" (McFarlane 2010: 732).

Um innerhalb dieser Vergleichskonstellation einen analytischen Raum jenseits des westzentrischen Diskurses vom säkularen Charakter urbaner Modernität zu öffnen, wählten wir akteurszentrierte und praxistheoretische Ansätze, die darauf zielten, die „Binnenperspektive der Handelnden und die Erfahrungen, die ihr zugrunde liegen, zu rekonstruieren" (Schiffauer 2010: 27). Die Fallstudien führten lokal situierte Forschende durch, deren Fellowships global ausgeschrieben waren. Um die herrschende Geopolitik des Wissens nicht unbedacht zu reproduzieren, erfolgte ihre Vergabe nach der Prämisse, unterschiedliche, auch jenseits akademischer Institutionen erworbene Wissensformen und Kompetenzen wertzuschätzen, anstatt die Bewertungsmuster euroamerikanischer Akademien anzuwenden. Daraus resultierte eine heterogene Zusammensetzung und methodische Experimentierfreudigkeit der Forschenden.

Das Konzept des Vergleichs als Strategie manifestierte sich in weiteren Manövern: In einer Öffnung für künstlerische Forschungsansätze etablierten wir Forschung als „procedure of exploration and discoveries, a constant and delicate movement between knowing and not-knowing" (Huffschmid 2012: 165f.). Wir konfigurierten das Projekt als transinstitutionelle Kooperation zwischen wissenschaftlichen, kulturellen, künstlerischen und politischen Institutionen und entwickelten das Wissen kollaborativ in Workshops und Forschungsexkursionen mit Partner_innen in den Untersuchungsstädten. Die Kollaboration von Wissenschaft und Kunst als je eigenständige epistemische Praktiken (Bippus 2009) sollte die gegenseitigen Ordnungssysteme irritieren, nach denen beide ihr Wissen erzeugen. Auf diese Weise wollten wir reflexive Befragungen etwa von selbstverständlich erscheinenden Prämissen oder von Subjektivitätseffekten der Forschenden 
fördern. Nicht zufällig, so die Überlegung, gingen in Deutschland die weitreichendsten Ansätze einer postkolonial informierten (Stadt-)Analyse wie „Projekt Migration“ (Kölnischer Kunstverein 2005), „Crossing Munich“ (Beyer et al. 2009) oder „Colonial Modern“(Avermaete et al. 2010) aus transdisziplinären akademisch-künstlerisch-aktivistischen Konstellationen hervor. Die Diversität der Kollaborationen generierte eine Perspektivenvielfalt bezogen auf Motive, Fragen und Methoden der Forschung. Kulturelle Inszenierungen, die den laufenden Forschungsprozess öffentlich präsentierten (vgl. www.globalprayers.info), ermöglichten die Reflexion seiner Strategien und Methoden in speziellen Diskursformaten. Beispielsweise diskutierten projektbeteiligte Forscher_innen, Protagonist_innen und Kritiker_innen in per Kopfhörer übertragenen Zweiergesprächen selbstkritisch ihre Möglichkeiten und Grenzen, um den Prämissen einer postkolonialen Kritik im Forschungsprozess gerecht zu werden.

Die komplexe Projektkonstellation, die Divergenz von Ordnungssystemen beteiligter Institutionen und Disziplinen, die Vielzahl von inhaltlichen Fallstudien und Fragen, die Überlagerung verschiedener Wissens- und Forschungstraditionen sowie die hohen Reflexivitätsansprüche erzeugten zahlreiche Konflikte, die im Forschungsprozess ausbalanciert werden mussten, und auch eine Reihe ungelöster Probleme. Beispielsweise existierten je nach lokaler Verortung der Forschenden (im Norden/Süden) hochgradig ungleiche Zugänge zu wichtigen Ressourcen (wie Bibliotheken oder universitäre Infrastrukturen). Im Rahmen der akademischen und geografischen Verortung des Projekts (in Berlin) war es nur sehr bedingt durchzuhalten, Methoden und Repräsentationsformen des erzeugten Wissens, die nicht den standardisierten Anforderungsprofilen der jeweiligen Disziplinen entsprachen, den erforderlichen Freiraum oder angemessenen Wert zu geben. Die Forschenden sahen sich gezwungen, den internationalen Standards ihrer künstlerischen oder wissenschaftlichen Disziplin gerecht zu werden, um ihre Karrieren nicht zu gefährden. Und aus dem Spannungsfeld zwischen der experimentellen Konstellation des Projekts und den Erwartungen der es finanzierenden Institutionen, die dem diametral entgegengesetzten Exzellenzdiskurs folgten, resultierte ein hoher Druck auf die Steuerung des Vorhabens und ein dauerhafter Konfliktherd, der zu einer negativen Zwischenevaluation und damit fast zum vorzeitigen Aus des Projekts führte.

\section{Was eine Postkolonialisierung der Stadtforschung (un-)möglich macht}

Um die Stadtforschung zu postkolonialisieren, ist es zwar weiter unabdingbar, den westzentrischen Charakter ihrer Prämissen zu dekonstruieren und theoretische Ansätze fortzuentwickeln, die postkoloniale Kritik reflektieren. Theorien aus dem Süden in der Stadt des globalen Nordens anzuwenden, kann aber nur ein erster Schritt sein. Für eine grundlegende Erneuerung im Sinne einer kosmopolitischeren und demokratischeren Stadtforschung ist es erforderlich, Forschung im Sinne eines Dialogprozesses zu etablieren, der postkoloniale Machtungleichheiten reflektiert und nicht nur verschiedene Disziplinen umfasst, sondern auch nichtakademische und aktivistische Formen der Wissensproduktion. Ein solches Forschen lässt sich fragend 
und mit offenem Ausgang auf eine Begegnung ein, „bei der wechselseitig Blicke und Reden ausgetauscht werden" (Berkin/Kaltmeier 2012: 10). Filip de Boeck drückte es auf dem Global-Prayers-Kongress so aus: „For me, decolonizing means: how can you invent a language about an encounter or describe a reality that you yourself are inevitably part of?"

Da eine als dialogischer Prozess konzipierte Forschung permanent „die bestehenden Normen, institutionalisierten Praktiken und Wissensformen“ infrage stellt (ebd.: 13), müssen die etablierten Komfortzonen der westlichen Universitäten überschritten werden. Dazu gehört es, neben den inhaltlichen und methodischen Paradigmen auch Akteurskonstellationen, Repräsentationsformen oder Evaluierungsweisen eines Forschungsprozesses auf eurozentrische Muster zu prüfen, das heißt dessen gesamte Einbettungsformen in die globale, von nachkolonialen Machtungleichheiten geprägte „Geopolitik des Wissens“(Walter Mignolo). Dies kann nur gelingen, wenn das klassische, an die Akademien gekoppelte Verständnis von Forschung und Wissen transdisziplinär und transinstitutionell geöffnet wird. Auch ist es erforderlich, sich Themen und Fragen - wie Religion, Gewalt etc. - zu stellen, die aus der Perspektive der kritischen Stadtforschung eingeübte Gewissheiten irritieren und daher zunächst unangenehm sind.

Zudem gilt auch hier, was Alexa Färber in ihrem Debattenbeitrag über urbane Assemblageforschung in $\mathrm{s} \mathrm{u} b \backslash \mathrm{u} \mathrm{r} \mathrm{b}$ a $\mathrm{n}$ bereits formuliert hat: Die geschilderten Anforderungen an eine Projektkonstellation bezogen auf mögliche Wege, Stadtforschung zu postkolonialisieren, sind „inkompatibel mit den Entwicklungen wissenschaftlicher Institutionen“ (2014: 101). Denn sie widersprechen deren Mainstreaming- und Homogenisierungsprozessen im Zuge der herrschenden Prämissen des ,Exzellenz'-Diskurses, der vereinheitlichten und quantifizierten Evaluierungs- und Konkurrenzlogik sowie der Marktfähigkeit des erzeugten Wissens. Zwar gilt dies für kritische Forschung generell. Um eine Postkolonialisierung voranzutreiben, genügt es aber nicht, die herkömmliche Forderung nach einer ,,analytischen Unabhängigkeit von Forschung" (ebd.: 102) zu erneuern. Vielmehr bezieht sich die Forderung, die westliche Stadtforschung von ihren Fundamenten aus zu dekonstruieren, auch auf deren kritische Varianten, die allzu oft ebenso blind für den westzentrischen Charakter ihrer Paradigmen sind.

\section{Endnoten}

[1] Bezeichnenderweise konzentrieren sich die BMBF-Projekte unter dem Label „Future Megacities“ mit dem Ziel, „technologische, soziale und wirtschaftliche Effizienzsprünge und ,Hebel' für Energieeffizienz und Klimaschutz zu erproben“ sowie „Lösungsstrategien“ zu entwickeln, die als „Modell auch für andere Megastädte dienlich sein“ sollen (BMBF 2010, Grußwort), in neokolonialistischer Manier ausschließlich auf Städte im globalen Süden. Die Tatsache, dass dabei auch in ihrer Größe und Bedeutung überschaubare Städte wie Casablanca, Hyderabad, Urumqui oder Addis Abeba unter das Label ,Megastadt“ fallen, verdeutlicht, dass sich ,Mega' weniger auf „,besonders groß, mächtig, hervorragend, bedeutend“ (Duden) bezieht, sondern dass eher die auch den Westen bedrohenden Dimensionen der Probleme und Fragen der Regierbarkeit von Städten das maßgebliche Kriterium dafür zu sein scheinen.

[2] Einen ähnlichen inhaltlichen Fokus hatte in dieser Zeitschrift der Debattenbeitrag von Margit Mayer (2013) über „urbane soziale Bewegungen in der neoliberalisierenden Stadt“. 
Aus meiner Sicht werden dabei aber die Thesen in der klassischen Manier der neomarxistischen Stadtforschung universalisiert, obwohl die Theorieansätze und Analysen des Beitrags unverkennbar nur auf urbane Konstellationen und Bewegungen in der euroamerikanischen Stadt sinnvoll anwendbar sind.

[3] Dagegen steht allerdings ein schockierender Zynismus, mit dem der rot-schwarze Berliner Senat in Person seines Innensenators mit einem juristischen Trick alle vertraglich fixierten politischen Zusagen an die Flüchtlingsaktivist_innen gebrochen hat, die er im Gegenzug für deren ,freiwillige‘ Räumung des Oranienplatzes gemacht hatte.

\section{Autor_innen}

Stephan Lanz betreibt Stadtforschung bevorzugt an disziplinären und institutionellen Schnittstellen zwischen Wissenschaften, Kultur und Aktivismus.

lanz@europa-uni.de

\section{Literatur}

Aktionskreis (2013): Unabhängiger Aktionskreis Non-Citizen Kämpfe: Ankündigung der Auflösung. Erklärung, publiziert am 9.9.2013. Online unter http://www.refugeetentaction.net/index.php?option $=$ com_content\&view=article\&id=272:aktionskreisunabhaengiger-non-citizen-kaempfe-ankuendigung-der-aufloesung\&catid=2\&Itemid=1 32\&lang=de (letzter Zugriff am 2.12.2014).

AlSayyad, Nezar (Hg.) (1992): Forms of Dominance. On the Architecture and Urbanism of the Colonial Enterprise. London/Aldershot: Avebury.

Appadurai, Arjun (2001): Deep democracy. Urban governmentality and the horizon of politics. In: Environment \& Urbanization 13/2, 23-44.

Appadurai, Arjun (2011): Cosmopolitanism from below. Some ethical lessons from the slums of Mumbai. Johannesburg Workshop of Theory and Criticism. The Salon 4. http://jwtc. org.za/volume_4/arjun_appadurai.htm (letzter Zugriff am 23.10. 2013).

Avermaete, Tom / Karakayali, Serhat / von Osten, Marion (Hg.) (2010): Colonial Modern. Aesthetics of the Past Rebellions of the Future. London: Blackdog Publishing.

Bayat, Asef (2012): Leben als Politik. Wie ganz normale Leute den Nahen Osten verändern. Berlin: Assoziation A.

Becker, Jochen / Burbaum, Claudia / Kaltwasser, Martin / Köbberling, Folke / Lanz, Stephan / Reichard, Katja (Hg.) (2003): Learning from*. Städte von Welt, Phantasmen der Zivilgesellschaft, informelle Organisation. Berlin: Vice Versa, metroZones 2.

Becker, Jochen / Klingan, Katrin / Lanz, Stephan / Wildner, Kathrin (Hg.) (2013): Global Prayers. Contemporary Manifestations of the Religious in the City. Zürich: Lars Müller Publishers, metroZones 13 .

Becker, Jochen / Lanz, Stephan (Hg.) (2003): Space//Troubles. Jenseits des Guten Regierens: Schattenglobalisierung, Gewaltkonflikte und städtisches Leben. Berlin: b_ books, metroZones 1.

Berkin, Sarah Corona / Kaltmeier, Olaf (2012): Im Dialog. Methodologische Überlegungen zu Horizontalität und Reziprozität in den Sozial- und Kulturwissenschaften. In: dies. (Hg.): Methoden dekolonisieren. Eine Werkzeugkiste zur Demokratisierug der Sozialund Kulturwissenschaften. Münster: Westfälisches Dampfboot, 7-16.

Beyer, Natalie / Engl, Andrea / Hess, Sabine / Moser, Johannes (Hg.) (2009): Crossing Munich. Beiträge zur Migration aus Kunst, Wissenschaft und Aktivismus. München: Silke Schreiber Verlag.

Bippus, Elke (Hg.) (2009): Kunst des Forschens. Praxis eines ästhetischen Denkens. Berlin/ Zürich: Diaphanes.

BMBF (2010): Megastädte - Die Welt von morgen nachhaltig gestalten. Bonn/Berlin: BMBF.

Castro Varela, María do Mar / Dhawan, Nikita (2005): Postkoloniale Theorie. Eine kritische Einführung. Bielefeld: transcript. 
Chakrabarty, Dipesh (2000): Provincialising Europe. Postcolonial Thought and Historical Difference. Princeton/Oxford: University of Princeton Press.

Chatterjee, Partha (2004): The Politics of the Governed. Reflections on Popular Politics in Most of the World. New York: Columbia University Press.

Clifford, James (1986): Introduction: Partial Truths. In: ders. / George Marcus (Hg.): Writing Culture. The Politics and Poetics of Ethnography. Berkeley u. a.: University of California Press, 1-26.

Comaroff, Jean / Comaroff, John (2012): Der Süden als Vorreiter der Globalisierung. Neue postkoloniale Perspektiven. Frankfurt a. M./New York: Campus.

Costa, Sérgio (2005): Postkoloniale Soziologie. Differenzen und Konvergenzen. In: Berliner Journal für Soziologie 2, 282-294.

De Boeck, Filip (2002): Kinshasa: tales of the 'invisible city' and the second world. In: Okwui Enwezor (Hg.): Under Siege: Four African Cities. Freetown, Johannesburg, Kinshasa, Lagos. Ostfildern: Hatje Cantz Verlag, 243-85.

Eckardt, Frank / Hoerning, Johanna (2012): Postkoloniale Städte. In: Frank Eckardt (Hg.): Handbuch Stadtsoziologie. Wiesbaden: Springer VS, 263-288.

Edensor, Tim / Jayne, Mark (Hg.) (2012): Urban Theory Beyond the West. A World of Cities. London/New York: Routledge.

Färber, Alexa (2014): Potentiale freisetzen. Akteur-Netzwerk-Theorie und Assemblageforschung in der interdisziplinären kritischen Stadtforschung. In: s u b $\backslash$ u r b a n. Zeitschrift für kritische Stadtforschung 2/1, 95-103.

Geographisches Institut der Universität Köln (o. D.): DFG-Schwerpunktprogramm Megacities - Megachallenge. Informal Dynamics of Global Change. http://www. geographie.uni-koeln.de/megacities-megachallenge.350.de.html (letzter Zugriff am 2.12.2014).

Gidwani, Vinay (2006): Subaltern cosmopolitanism as politics. In: Antipode 38, 7-21.

Ha, Noa (2014): Perspektiven urbaner Dekolonisierung. Die europäische Stadt als ,Contact Zone'. In: s u b $\backslash$ u r b a n. Zeitschrift für kritische Stadtforschung 2/1, 27-47.

Hall, Stuart (1994): Rassismus und kulturelle Identität. Ausgewählte Schriften 2. Hamburg: Argument.

Heck, Gerda / Lanz, Stephan (2014): Religiöses ,worlding in der Stadt: Globaler Pentekostalismus in Rio de Janeiro. In: Peripherie. Zeitschrift für Politik und Ökonomie in der Dritten Welt 134/135, 212-238.

Hess, Sabine / Lebuhn, Henrik (2014): Politiken der Bürgerschaft. Zur Forschungsdebatte um Migration, Stadt und citizenship. In: $\mathrm{s} \mathrm{u} \mathrm{b} \backslash$ u r b a n. Zeitschrift für kritische Stadtforschung 2/3, 11-34.

Huffschmid, Anne (2012): Another way of knowing. Notes on visual research on ghosts and spirits. In: metroZones (Hg.): Faith is the Place. The Urban Cultures of Global Prayers. Berlin: b_books, metroZones 11, 164-175.

Huffschmid, Anne / Wildner, Kathrin (2013): Stadtforschung aus Lateinamerika. Neue urbane Szenarien: Öffentlichkeit - Territorialität - Imaginarios. Bielefeld: transcript.

Isin, Engin F. (2003): Historical sociology of the city. In: Gerard Delanty / ders. (Hg.): Handbook of Historical Sociology. London u. a.: Sage, 312-325.

Isin, Engin F. (2008): Theorizing acts of citizenship. In: ders. / Greg M. Nielsen (Hg.): Acts of Citizenship. London: Palgrave Macmillan, 15-43.

Jacobs, Jane M. (1996): Edge of Empire. Postcolonialism and the City. London/New York: Routledge.

Jacobs, Jane M. (2012): Commentary: Comparing comparative urbanisms. In: Urban Geography 33/ 6, 904-914.

Kaltmeier, Olaf (2012): Methoden dekolonisieren. Reziprozität und Dialog in der herrschenden Geopolitik des Wissens. In: ders. / Sarah Corona Berkin (Hg.): Methoden dekolonisieren. Eine Werkzeugkiste zur Demokratisierug der Sozial- und Kulturwissenschaften. Münster: Westfälisches Dampfboot, 18-44.

King, Anthony (1990): Urbanism, Colonialism, and the World Economy. Cultural and Spatial Foundations of the World Urban System. London/New York: Routledge.

King, Anthony (2003): Postcolonialism, representation, and the city. In: Gary Bridge / Sophie Watson (Hg.): A Companion to the City. Malden/Oxford: Blackwell Publishers, 261-269.

Kölnischer Kunstverein (Hg.) (2005): Projekt Migration. Köln: DuMont. 
Kotti \& Co (2012): Merhaba, Salam und schönen guten Tag. http://kottiundco. net/2012/08/o3/merhaba-salam-und-schonen-guten-tag (letzter Zugriff am 15.1. 2014).

Lanz, Stephan (2007): Berlin aufgemischt: abendländisch - multikulturell - kosmopolitisch? Die politische Konstruktion einer Einwanderungsstadt. Bielefeld: transcript.

Lanz, Stephan (2008): ,In Europa mehr Initiative und Kraft entwickeln‘. Herrschaftsverhältnisse im globalen Städtesystem des Postkolonialismus. In: Sophie Wolfrum / Susanne Schaubeck (Hg.): Multiple City. Citykonzepte 1908/2008. Berlin: Jovis, 294-298.

Lanz, Stephan (2013): Berlin diversities. The perpetual act of becoming a true metropolis. In: Matthias Bernt / Britta Grell / Andrej Holm (Hg.): The Berlin Reader. Bielefeld: transcript, 209-224.

Lanz, Stephan (2014a): Assembling global prayers in the city. An attempt to repopulate urban theory with religion. In: Jochen Becker / Katrin Klingan / Stephan Lanz / Kathrin Wildner (Hg.): Global Prayers. Contemporary Manifestations of the Religious in the City. Zürich: Lars Müller Publishers, 16-47.

Lanz, Stephan (2014b): Politik zwischen Polizei und Post-Politik. Überlegungen zu ,urbanen Pionieren' einer politisierten Stadt am Beispiel von Berlin. In: Melanie Behrens / WolfDietrich Bukow / Karin Cudak (Hg.): Inclusive City. Überlegungen zum gegenwärtigen Verhältnis von Mobilität und Diversität in der Stadtgesellschaft. Wiesbaden: Springer VS, im Erscheinen.

Lossau, Julia (2012): Postkoloniale Impulse für die deutschsprachige Geographische Entwicklungsforschung. In: Geographica Helvetica 67, 125-132.

Mayer, Margit (2013): Urbane soziale Bewegungen in der neoliberalisierenden Stadt. In: $\mathrm{s}$ u b $\backslash \mathrm{u}$ r b a n. Zeitschrift für kritische Stadtforschung 1/1, 155-168.

McFarlane, Colin (2010): The comparative city. Knowledge, learning, urbanism. In: International Journal of Urban and Regional Research 34/4, 725-42.

Mecheril, Paul (2004): Einführung in die Migrationspädagogik. Weinheim/Basel: Beltz.

Michel, Boris / Roskamm, Nicolai (2013): Einführung: Die postpolitische Stadt. In: s u b \ u r b a n. Zeitschrift für kritische Stadtforschung 1/2, 9-16.

Mignolo, Walter (200o): The many faces of cosmo-polis. Border thinking and critical cosmopolitanism. In: Public Culture 12, 721-748.

Mignolo, Walter (2002): The geopolitics of knowledge and the colonial difference. In: The South Atlantic Quarterly 10/1, 57-96.

Ong, Aihwa (2011): Worlding cities, or the art of being global. In: Ananya Roy / dies. (Hg.): Worlding Cities. Asian Experiments and the Art of Being Global. Malden/Oxford: Blackwell Publishers, 1-26.

Rancière, Jacques (2002): Das Unvernehmen. Politik und Philosophie. Frankfurt a. M.: Suhrkamp.

Robinson, Jennifer (2006): Ordinary Cities. Between Modernity and Development. London/ New York: Routledge.

Robinson, Jennifer (2010): Cities in a world of cities. The comparative gesture. In: International Journal of Urban and Regional Research 35/1, 1-23.

Roy, Ananya (2009): The 21st-century metropolis. New geographies of theory. In: Regional Studies 43/6, 819-30.

Roy, Ananya (2011a): Slumdog cities. Rethinking subaltern urbanism. In: International Journal of Urban and Regional Research 35/2, 223-38.

Roy, Ananya (2011b): Postcolonial urbanism. Speed, hysteria, mass dreams. In: dies. / Aihwa Ong (Hg.): Worlding Cities. Asian Experiments and the Art of Being Global. Malden/ Oxford: Blackwell Publishers, 307-35.

Roy, Ananya (2014): Worlding the south. Toward a post-colonial urban theory. In: Susan Parnell / Sophie Oldfield (Hg.): The Routledge Handbook on Cities of the Global South. London/New York: Routledge, 9-20.

Roy, Ananya / Ong, Aihwa (Hg.) (2011): Worlding Cities. Asian Experiments and the Art of Being Global. Malden/Oxford: Blackwell Publishers.

Schiffauer, Werner (2010): Nach dem Islamismus. Eine Ethnographie der Islamischen Gemeinschaft Milli Görüş. Frankfurt a. M.: Suhrkamp.

Siebel, Walter (2012): Die europäische Stadt. In: Frank Eckardt (Hg.): Handbuch Stadtsoziologie. Wiesbaden: Springer VS, 201-211.

Simone, AbdouMaliq (2001): On the worlding of African cities. In: African Studies Review 44/2, 15 -41. 
Simone, AbdouMaliq (2010): City Life from Jakarta to Dakar. Movements at the Crossroads. London/New York: Routledge.

Simone, AbdouMaliq (2011): The surfacing of urban life. In: City 15/3-4, 355-364.

Spivak, Gayatri Chakravorty (1999): A Critique of Postcolonial Reason. Toward a History of the Vanishing Present. Cambridge/London: Harvard University Press.

Tamayo, Sergio (2013): Die Stadt und die Produktion des Ciudadanía-Raums. Eine stadtsoziologische Perspektive. In: Anne Huffschmid / Kathrin Wildner (Hg.): Stadtforschung aus Lateinamerika. Neue urbane Szenarien: Öffentlichkeit - Territorialität - Imaginarios. Bielefeld: transcript, 203-223.

Terkessidis, Mark (2004): Die Banalität des Rassismus. Migranten zweiter Generation entwickeln eine neue Perspektive. Bielefeld: transcript.

Tsianos, Vassilis (2013): Urbane Paniken. Zur Entstehung eines antimuslimischen Urbanismus. In: Duygu Gürsel / Zülfukar Çetin / Allmende e. V. (Hg.): Wer MACHT Demo_kratie? Kritische Beiträge zu Migration und Machtverhältnissen. Münster: edition assemblage, 22-42.

Yeoh, Brenda S. A. (2001): Postcolonial cities. In: Progress of Human Geography 25/3, 456-468.

Ziai, Aram (2012): Post-Development. Fundamentalkritik der „Entwicklung“. In: Geographica Helvetica 67, 133-138.

\section{On (im-)possibilities to postcolonialize western urban studies}

Taking as a starting point that western urban theory is based on eurocentric premises connecting urban modernity to western cities and subjecting cities elsewhere to the developmentalism of catching up with modernization, this paper argues for the necessity of postcolonializing urban studies. Using two examples, the article discusses possible ways of promoting theoretical and methodological approaches that reflect postcolonial critique. On the one hand, 'theories of the south' are applied in order to shake established truths about the city of the global North. On the other hand, the international research project "Global Prayers - Redemption and Liberation in the City" is discussed as an attempt to deconstruct some of the eurocentric fundaments of urban studies and to promote more cosmopolitan urban studies with the help of a transregional, transdisciplinary and transinstitutional approach. 
